\title{
Extra-virgin olive oil consumption improves the capacity of HDL to mediate cholesterol efflux and increases $A B C A 1$ and $A B C G 1$ expression in human macrophages
}

\author{
Olfa Helal $^{1}$, Hicham Berrougui ${ }^{1}$, Soumaya Loued $^{1}$ and Abdelouahed Khalil ${ }^{1,2 *}$ \\ ${ }^{1}$ Research Center on Aging, 1036 rue Belvédère Sud, Sherbrooke, QC, Canada J1H 4C4 \\ ${ }^{2}$ Department of Medicine, Geriatrics Service, Faculty of Medicine, University of Sherbrooke, Sherbrooke, QC, Canada \\ (Submitted 30 March 2012 - Final revision received 26 July 2012 - Accepted 31 July 2012 - First published online 10 October 2012)
}

\begin{abstract}
The present study was aimed to investigate the effect of 12 weeks of extra-virgin olive oil (EVOO) consumption on the capacity of HDL to promote cholesterol efflux (CE) and to determine which CE pathways are modulated by EVOO consumption. Whole $\mathrm{HDL}_{\text {and }} \mathrm{HDL} / \mathrm{HDL}_{3}$ subclasses were isolated from the plasma of twenty-six healthy volunteers before and after 12 weeks of EVOO consumption ( $25 \mathrm{ml} / \mathrm{d})$. EVOO consumption increased the capacity of serum and HDL to mediate CE from THP-1, J774 macrophages and Fu5AH cells by 9.8-24.57\%, depending on the cell type. The increase in CE was independent of both HDL concentration and subclass distribution. The three HDL-mediated CE pathways (ATP-binding cassette (ABC) A1, ABCG1 and scavenger receptor class B type I (SR-BI)) were modulated by EVOO consumption. The fluidity of the phospholipidic layer of HDL increased by $13 \%(P<0 \cdot 001)$ following EVOO consumption compared with baseline. EVOO consumption also increased the release of excess cholesterol from human monocyte-derived macrophages (HMDM) by $44 \%(P<0 \cdot 001)$, and $A B C A 1$ and $A B C G 1$ mRNA transcription by $16 \cdot 08 \%(P<0 \cdot 001)$ and $35 \cdot 79 \%(P<0 \cdot 01)$, respectively. The protein expression of these two cholesterol transporters also increased after EVOO consumption. In contrast, SR-BI mRNA and protein expression in HMDM were significantly lower after 12 weeks of EVOO consumption. Incubating J774 macrophages with EVOO polyphenol extracts induced a concentration-dependent up-regulation of ABCA1 and ABCG1 expression in macrophages. After 12 weeks of EVOO consumption, the capacity of HDL to mediate CE was improved and the ability of HMDM to release excess cholesterol was enhanced by increasing the expression of ABCA1 and ABCG1 transporters.
\end{abstract}

Key words: HDL: Extra-virgin olive oil: Cholesterol efflux: ABCA1/ABCG1

The concentration of HDL is inversely associated with the incidence of CHD. HDL are thus considered anti-atherogenic lipoproteins $^{(1,2)}$. One of the major anti-atherogenic activities of HDL is the regulation of cholesterol homeostasis through the reverse cholesterol transport (RCT) process. RCT involves the transport of excess cholesterol from peripheral tissues back to the liver for elimination in the bile and faeces. The initial step in RCT is thought to be the transfer of cholesterol from cell membranes to acceptor particles and then to apoA$\mathrm{I}$ and $\mathrm{HDL}^{(3-5)}$

HDL-mediated cholesterol efflux (CE) is the natural rate-limiting step of $\mathrm{RCT}^{(6)}$ and occurs via three pathways. The first is aqueous diffusion by which free cholesterol molecules spontaneously desorb from the plasma membrane, diffuse through the aqueous phase and become adsorbed on acceptor particles by collision ${ }^{(7)}$. The second involves scavenger receptor class B type I (SR-BI)-mediated bidirectional free cholesterol exchanges that depend on the cholesterol gradient. This pathway mediates CE to a wide variety of cholesterol acceptors $^{(8)}$. The third involves the ATP-binding cassette receptors $\mathrm{ABCA} 1$ and $\mathrm{ABCG} 1$, which mediate $\mathrm{CE}$ in a unidirectional manner to lipid-poor apoA-I and to other subfamily members of HDL, respectively ${ }^{(9-11)}$. The capacity of HDL to mediate CE depends on their biophysical structure and biochemical composition. For example, phosphatidylcholineenriched HDL increases CE, whereas sphingomyelin-enriched HDL decreases cholesterol influx to macrophages ${ }^{(12)}$.

A large body of literature has indicated that diet is one of the most important modifiable determinants of the risk of developing $\mathrm{CVD}^{(13,14)}$. Replacement of dietary SFA with higher

Abbreviations: ABC, ATP-binding cassette; CE, cholesterol efflux; DMEM, Dulbecco's modified Eagle's medium; DPH, 1,6-diphenyl-1,3,5-hexatriene; EVOO, extra-virgin olive oil; FBS, fetal bovine serum; HMDM, human monocyte-derived macrophages; RCT, reverse cholesterol transport; RPMI, Roswell Park Memorial Institute; SR-BI, scavenger receptor class B type I.

*Corresponding author: Dr A. Khalil, fax +1 819829 7141, email abdelouahed.khalil@usherbrooke.ca 
intakes of MUFA from vegetable oils has been reported to be inversely associated with the risk of $\mathrm{CHD}^{(15)}$.

Olive oil is the main source of fat in Mediterranean regions. The alleged beneficial effects of extra-virgin olive oil (EVOO) have been linked to both its MUFA (mainly oleic acid) and its antioxidant components (e.g. hydroxytyrosol and oleuropein). Olive oil phenolics exert their antioxidant effect both in vitro and in vivo and have potential cardioprotective activities (reviewed in Visioli et al $^{(16)}$ ). Considerable attention is being paid to the potential health benefits of olive oil. Human consumption of olive oil decreases the major risk factors associated with atherosclerosis by improving the lipoprotein profile, blood pressure, glucose metabolism, oxidative stress and thrombotic profiles (reviewed in Lopez-Miranda et $a l^{(17)}$ ). Olive oil supplementation also protects against LDL and HDL oxidation ${ }^{(18-20)}$ and reduces total cholesterol and LDL-cholesterol levels ${ }^{(21)}$. Several studies have investigated the effect of olive oil consumption on plasma HDL levels and have led to controversial results ${ }^{(22-25)}$. However, no studies have investigated the effect of olive oil consumption on the functionality of HDL and, particularly, on their capacity to mediate CE. However, it is becoming increasingly apparent that the anti-atherogenic effects of HDL not only depend on their concentration and cholesterol content in the circulatory system, but also, and more importantly, on their biological functionalities ${ }^{(26)}$. Furthermore, studies have reported that diet, such as supplementation with MUFA in human subjects, influences lipoprotein functionality by enhancing HDL-mediated $\mathrm{CE}^{(27,28)}$ and that EVOO consumption increases the phenolic compound content of LDL and HDL, which prevents lipid peroxidation and promotes the antiatherogenic effect of $\mathrm{HDL}^{(28)}$.

The goals of the present study were to investigate the effect of 12 weeks of EVOO consumption on the capacity of HDL to promote $\mathrm{CE}$ and to determine which CE pathways are modulated by EVOO consumption.

\section{Materials and methods}

\section{Chemicals}

SDS, EDTA and bovine serum albumin were from Sigma. Dialysis membranes were from Spectrum Medical Industries, Inc. Dulbecco's modified Eagle's medium (DMEM) and fetal bovine serum (FBS) were from Wisent, Inc. All other chemicals were from Sigma-Aldrich. THP-1 cells and J774 macrophages were from the American Type Culture Collection. Fu5AH rat hepatoma cells were provided by Dr J. Genest (McGill University). Roswell Park Memorial Institute (RPMI) 1640 medium was from Invitrogen Canada, Inc. EVOO was from Atlas Olive Oil sarl.

\section{Subjects}

A total of twenty-six healthy volunteers aged between 25 and 83 years (eight men and eighteen women, mean age 53.28 (sEm 20.66) years) were recruited. They were all non-smokers with a normal serum lipid profile and blood pressure and were not taking medication, including lipid-lowering drugs or oral antioxidants. Both pre- and postmenopausal women were included in the study. However, none of the women was taking oestrogen replacement therapy for menopause. They were all non-obese and none showed clinical signs of inflammation or diabetes. The clinical and biochemical parameters of the volunteers are presented in Table 1.

The present study was conducted according to the guidelines laid down in the Declaration of Helsinki and all procedures involving human subjects/patients were approved by the Ethics Committee of the University Institute of Geriatrics of Sherbrooke (no. 2009/19). Written informed consent was obtained from all subjects.

\section{Study design and procedure}

The volunteers were asked to consume $25 \mathrm{ml} / \mathrm{d}$ of raw EVOO for 12 weeks. The chemical composition of EVOO used in the study is described in Table $2^{(29)}$. Blood tests were performed at recruitment (T0) and after 12 weeks of EVOO consumption (T12). Plasma tyrosol and hydroxytyrosol levels were measured by HPLC, using the diode-array UV detection method as developed by Ruiz-Gutierrez et al. ${ }^{(30)}$, to evaluate compliance.

\section{Lipoprotein isolation}

Fasting human plasma was collected in heparin-containing tubes. Whole $\mathrm{HDL}$ and $\mathrm{HDL}_{2} / \mathrm{HDL}_{3}$ subclasses were isolated

Table 1. Clinical and biochemical parameters of the participants at baseline and after 12 weeks of extra-virgin olive oil consumption (Mean values with their standard errors or standard deviations)

\begin{tabular}{|c|c|c|c|c|}
\hline & \multicolumn{2}{|c|}{ T0 } & \multicolumn{2}{|c|}{ T12 } \\
\hline & Mean & SEM & Mean & SEM \\
\hline$n$ & \multicolumn{2}{|c|}{26} & & \\
\hline Women & \multicolumn{2}{|c|}{18} & & \\
\hline Men & \multicolumn{2}{|c|}{8} & & \\
\hline \multicolumn{5}{|l|}{ Age (years) } \\
\hline Mean & \multicolumn{2}{|c|}{$20 \cdot 66$} & & \\
\hline SD & \multicolumn{2}{|c|}{$53 \cdot 28$} & & \\
\hline BMI $\left(\mathrm{kg} / \mathrm{m}^{2}\right)$ & $24 \cdot 9$ & 0.65 & 24.9 & 0.59 \\
\hline Systolic blood pressure $(\mathrm{mmHg})$ & 126 & $3 \cdot 10$ & 123 & $2 \cdot 4$ \\
\hline Diastolic blood pressure $(\mathrm{mmHg})$ & 77 & 1.65 & 77 & $1 \cdot 35$ \\
\hline $\mathrm{TC}(\mathrm{mmol} / \mathrm{l})$ & $5 \cdot 10$ & $0 \cdot 16$ & 4.94 & 0.019 \\
\hline TAG (mmol/l) & $1 \cdot 27$ & 0.13 & $1 \cdot 16$ & 0.11 \\
\hline HDL-C (mmol/l) & 1.50 & 0.07 & 1.50 & 0.06 \\
\hline LDL-C (mmol/l) & 3.03 & 0.13 & 2.91 & 0.15 \\
\hline ApoA1 (g/l) & 1.60 & 0.05 & 1.59 & 0.04 \\
\hline ApoB (g/l) & 0.90 & 0.04 & 0.89 & 0.04 \\
\hline ApoB:apoA1 & 0.80 & 0.04 & 0.84 & 0.04 \\
\hline TC:HDL-C & 3.58 & 0.19 & $3.43^{*}$ & 0.38 \\
\hline LDL-C:HDL-C & $2 \cdot 25$ & 0.14 & $2 \cdot 01$ & 0.16 \\
\hline TAG:HDL-C & 0.94 & 0.12 & $0.84^{*}$ & 0.10 \\
\hline Glucose (mmol/l) & 4.42 & 0.10 & $4 \cdot 44$ & 0.10 \\
\hline Insulin (pmol/l) & $35 \cdot 20$ & 4.01 & $37 \cdot 63$ & 3.98 \\
\hline CRP (mg/l) & 3.43 & 0.26 & $3 \cdot 48$ & 0.30 \\
\hline
\end{tabular}


Table 2. Chemical composition of extra-virgin olive oil (EVOO) used in the study ${ }^{(29)}$

\begin{tabular}{lc}
\hline & EVOO \\
\hline Fatty acids (\%) & \\
$16: 0$ & $10 \cdot 4$ \\
$18: 0$ & $2 \cdot 76$ \\
$18: 1 n-9$ & $71 \cdot 0$ \\
$18: 2 n-6$ & $12 \cdot 9$ \\
$18: 3 n-3$ & $1 \cdot 04$ \\
Sterols (mg/100 g oil) & 319 \\
Schottenol & 0 \\
Spinasterol & 0 \\
Stigmasta-8,22-dien-3 $\beta$-ol & 0 \\
$\beta-S i t o s t e r o l$ & 156 \\
Campesterol & 12 \\
Others & 151 \\
Tocopherols (mg/kg oil) & 257 \\
$\alpha$ & 190 \\
$\delta$ & 42 \\
$\gamma$ & 26 \\
Phenolic compounds ( $\mu \mathrm{g} / \mathrm{kg}$ oil) & 792983 \\
Vanillic acid & 359 \\
Syringic acid & 0 \\
Ferulic acid & 51 \\
Tyrosol & 19573 \\
Others & 773000 \\
\hline
\end{tabular}

within 2 and $4 \mathrm{~h}$, respectively, using the method of Sattler et $a l^{(31)}$. HDL were stored in phosphate buffer containing $10 \%$ sucrose at $-80^{\circ} \mathrm{C}$ until used. Before the analysis, frozen HDL were dialysed overnight at $4^{\circ} \mathrm{C}$ in $10 \mathrm{~mm}$-sodium phosphate buffer ( $\mathrm{pH} 7 \cdot 0$ ) to remove sucrose, and protein concentrations were measured using commercial assay kits (Bio-Rad) according to the manufacturer's instructions.

\section{Cell cultures}

Human THP-1 monocytes and J774 macrophages were grown in RPMI-1640 medium and DMEM, respectively. The media were supplemented with $10 \%$ heat-inactivated FBS, $50 \mathrm{~nm}-\beta$-mercaptoethanol (only for THP-1), 2 mM-L-glutamine, $5 \mathrm{mg} / \mathrm{ml}$ of glucose and $100 \mathrm{U} / \mathrm{ml}$ of penicillin. THP- 1 monocytes were differentiated into macrophages by cultivating the cells in the presence of $100 \mu \mathrm{m}$-phorbol myristate acetate for 96h. Fu5AH hepatoma cells were grown in DMEM supplemented with $5 \%$ heat-inactivated FBS and $100 \mathrm{U} / \mathrm{ml}$ of penicillin-streptomycin.

\section{Human monocyte isolation and differentiation into macrophages}

Human monocytes were isolated as described previously ${ }^{(32)}$ and were differentiated into macrophages (human monocyte-derived macrophages (HMDM)). Briefly, peripheral blood mononuclear cells were isolated by density gradient centrifugation on Ficoll-Paque ${ }^{\mathrm{TM}}$ PLUS columns according to the manufacturer's instructions (GE Healthcare). Peripheral blood mononuclear cells were resuspended in 10\% FBS-RPMI medium, plated in twelve-well plates $\left(0.5 \times 10^{5}\right.$ cells per well $)$ pre-coated with $20 \%$ autologous serum, and were allowed to attach for $20 \mathrm{~min}$. Unattached cells were then removed from the medium. Attached monocytes were incubated with $10 \%$ FBS-RPMI containing $100 \mu \mathrm{m}$-phorbol myristate acetate to differentiate them into HMDM. The medium was changed every $2-3 \mathrm{~d}$. The monocytes differentiated into macrophages after $7 \mathrm{~d}$. The macrophages were used to assess $A B C A 1$, $A B C G 1$ and $S R-B I$ gene and protein expression at baseline (T0) and after 12 weeks of EVOO consumption (T12).

\section{Cholesterol efflux measurements}

THP-1, J774 and Fu5AH cells were incubated in fresh culture medium containing $0 \cdot 2 \mu \mathrm{Ci} / \mathrm{ml}$ of $\left[{ }^{3} \mathrm{H}\right]$ cholesterol for $24 \mathrm{~h}$. The cells were then washed and incubated in serumfree medium containing $1 \%$ bovine serum albumin for $12 \mathrm{~h}$ for equilibration following which they were washed and suspended in fresh medium without HDL (control), fresh medium containing $5 \%$ serum, fresh medium containing $50 \mu \mathrm{g} / \mathrm{ml}$ of whole HDL, or fresh medium containing $50 \mu \mathrm{g} / \mathrm{ml}$ of $\mathrm{HDL}_{3}$ or $\mathrm{HDL}_{2}$ obtained from each volunteer.

In another series of experiments, J774 macrophages were incubated for $12 \mathrm{~h}$ with $0.3 \mathrm{~mm}$-cyclic AMP to yield ABCA1-enriched macrophages. The cells were then washed and incubated with $5 \%$ serum, $\mathrm{HDL}_{2}$ or $\mathrm{HDL}_{3}$ for $24 \mathrm{~h}$. ABCG1 overexpression on THP-1 macrophages was induced by incubating phorbol myristate acetate-pretreated THP-1 macrophages with $5 \mu \mathrm{M}-22(R)$-hydroxy-cholesterol for $24 \mathrm{~h}^{(33)}$. RT-PCR and Western blot analyses were performed to confirm the overexpression of ABCG1 mRNA and protein, respectively.

HMDM were loaded with $\left[{ }^{3} \mathrm{H}\right]$ cholesterol $(1 \mu \mathrm{Ci} / \mathrm{ml})$ for $48 \mathrm{~h}$. Labelled HMDM were washed and incubated in serumfree medium containing $1 \%$ bovine serum albumin for $12 \mathrm{~h}$ for equilibration following which they were incubated for $4 \mathrm{~h}$ with $25 \mu \mathrm{g} / \mathrm{ml}$ of human apoA-I in order to measure CE.

$\mathrm{CE}$ was measured by liquid scintillation counting. The percentage of radiolabelled CE was calculated using the following formula:

$$
(\mathrm{cpm} \text { in medium } / \mathrm{cpm} \text { in cells }+ \text { medium }) \times 100,
$$

where cpm is counts per min.

\section{Agarose gel electrophoresis and Western blotting}

HDL subclasses were analysed by agarose gel electrophoresis. Aliquots of plasma and $\mathrm{HDL}_{2}$ and $\mathrm{HDL}_{3}$ particles obtained from the volunteers at T0 and T12 were separated on $0.75 \%$ agarose gels and transferred to a nitrocellulose membrane. ApoA-I was identified using an anti-apoA-I monoclonal primary antibody and an IgG-horseradish peroxidase secondary antibody. Immunocomplexes were visualised using an enhanced chemiluminescence Western blotting detection system followed by autoradiography.

HDL particle sizes were also measured by linear PAGE (Quantimetrix Lipoprint System; Quantimetrix). Overall, twelve plasma samples were kindly analysed by Quantimetrix to compare the effect of 12 weeks of EVOO consumption on the distribution of the HDL subclasses. The analysis was 
performed using polyacrylamide gel tubes and the Lipoprint LDL system (Quantimetrix) ${ }^{(34)}$.

\section{Quantitative PCR analyses}

RNA (2 $\mu \mathrm{g})$ extracted from HMDM (TRIzol; Invitrogen) were transcribed using Reverse Transcriptase Superscript II (Invitrogen). Quantitative PCR assays were performed using $25 \mathrm{ng}$ of template complementary DNA. The conditions for all the reactions were as follows: an initial 10 min denaturation step at $95^{\circ} \mathrm{C}$, followed by $40 \mathrm{~s}$ cycles at 95,56 and $72^{\circ} \mathrm{C}$. Quantitative PCR assays were performed using an Mx3005P ${ }^{\circledR}$ QPCR system (Agilent Technologies) and a Brilliant II SYBR Green QPCR Master Mix (Agilent Technologies). Results were calculated using the $2^{-\Delta \Delta C_{t}}$ relative quantification method normalised to $\beta$-actin. The primer sets are listed in Table 3. The comparative threshold cycle $\left(C_{\mathrm{t}}\right)$ method was used to quantify transcript levels and to normalise $\beta$-actin expression.

\section{Western blotting analyses}

To gain more insight into the effect of EVOO consumption on the modulation of $\mathrm{CE}, \mathrm{ABCA} 1, \mathrm{ABCG} 1$ and SR-B1 protein expression in HMDM were quantified by Western blot analysis at T0 and T12. Moreover, the expression of these proteins was also measured in $\mathrm{J} 774$ macrophages after incubation of these cells, for $12 \mathrm{~h}$, with EVOO total phenolic extract or with the major phenolic compounds of EVOO such as tyrosol and hydroxytyrosol at concentrations of 5 and $10 \mu \mathrm{m}$, respectively. In both experiments, lysate proteins were loaded on $10 \%$ acrylamide gels and separated by SDS-PAGE. The bands were transferred to nitrocellulose membranes, which were blocked with $5 \%$ skimmed milk in PBS/Tween-20. The blots were incubated with primary antibodies and then with specific IgG-horseradish peroxidase secondary antibodies. $\beta$-Actin was used as a control of protein loading. Protein bands were detected using an enhanced chemiluminescence reagent ${ }^{(35)}$.

\section{HDL fluidity measurements}

Lipoprotein fluidity was measured based on the steady-state anisotropy of 1,6-diphenyl-1,3,5-hexatriene (DPH) dissolved in tetrahydrofuran, as described previously ${ }^{(36)}$. Briefly, HDL were incubated with $1 \mu \mathrm{M}-\mathrm{DPH}$ for $15 \mathrm{~min}$ at $37^{\circ} \mathrm{C}$ in the dark with constant stirring. Steady-state fluorescent polarisation of DPH was measured at $37^{\circ} \mathrm{C}$ using a Hitachi spectrofluorometer model F-4500 (Hitachi, Limited). DPH was excited using a vertically polarised light at $360 \mathrm{~nm}$, and emission intensities were detected at $430 \mathrm{~nm}$ through a polariser orientated parallel and perpendicular to the direction of polarisation of the excitation beam. Steady-state fluorescence anisotropy ( $r$ ) was calculated using the following equation from the FL solution program (Hitachi):

$$
I_{\mathrm{v}}-G I_{\mathrm{p}} /\left(I_{\mathrm{v}}-2 G I_{\mathrm{p}}\right),
$$

where $I_{\mathrm{v}}$ and $I_{\mathrm{p}}$ are the parallel and perpendicular polarised fluorescence intensities, and $G$ is the monochromator grating correction factor. Fluidity is the inverse value of anisotropy and is expressed as $1 / r$ for steady-state fluorescence anisotropy.

\section{Statistical analysis}

Values are expressed as means with their standard errors. A Wilcoxon matched-pairs signed-rank test was used to compare differences between groups. Statistical analyses were performed using GraphPad Prism version 5.0 (GraphPad Software, Inc.).

\section{Results}

Table 1 presents the clinical and biochemical parameters of the volunteers at baseline (T0) and after 12 weeks of EVOO consumption (T12). The volunteers (mean age 53.28 (sEm 20.66) years) were healthy and non-obese (BMI 24.9 (SEM 0.65$) \mathrm{kg} / \mathrm{m}^{2}$ ) with lipid profiles and systolic and diastolic blood pressure in normal ranges. There were no significant differences in serum TAG, total cholesterol, HDL-cholesterol and LDL-cholesterol, and other cardiovascular risk markers between T0 and T12. The TAG:HDL and total cholesterol:HDL ratios, which are atherogenic indices, decreased respectively by $10.1 \%(P<0.05)$ and $4.2 \%(P<0.05)$ at T12. Plasma tyrosol and hydroxytyrosol contents showed a small (not statistically significant) increasing trend after 12 weeks of EVOO consumption (results not shown).

\section{Effect of extra-virgin olive oil consumption on serum- mediated cholesterol efflux}

CE is the first rate-limiting step of RCT and is influenced by the capacity of HDL to act as a cholesterol acceptor and of peripheral cells to release excess cholesterol.

We first investigated the effect of 12 weeks of EVOO consumption on the capacity of serum to mediate CE. As shown in Fig. 1(a), 12 weeks of EVOO consumption resulted in a

Table 3. Sequences of primers for real-time quantitative PCR

\begin{tabular}{lll}
\hline Genes & Forward primers $\left(5^{\prime}\right.$ to $\left.3^{\prime}\right)$ & Reverse primers $\left(5^{\prime}\right.$ to $\left.3^{\prime}\right)$ \\
\hline$A B C A 1$ & TCATCTTCATCTGCTTCCAGC & GTGCTGGGGATCTTGAACAC \\
$A B C G 1$ & GCCGTCGCTCATCAAGCA & GATAGGGATCTCCTTCCACATGTTG \\
SR-BI & TTCTTCTCCATGCTGTTCCTC & GCCAGGTAGTAGGCCTTCAG \\
$\beta-$ Actin & GAACGGTGAAGGTGACA & TAGAGAGAAGTGGGGTGG \\
\hline
\end{tabular}


$9 \cdot 8 \%(P<0 \cdot 01)$ increase in the capacity of serum to mediate CE from THP-1 macrophages.

We then investigated which CE pathway was stimulated the most after 12 weeks of EVOO consumption. We used J774

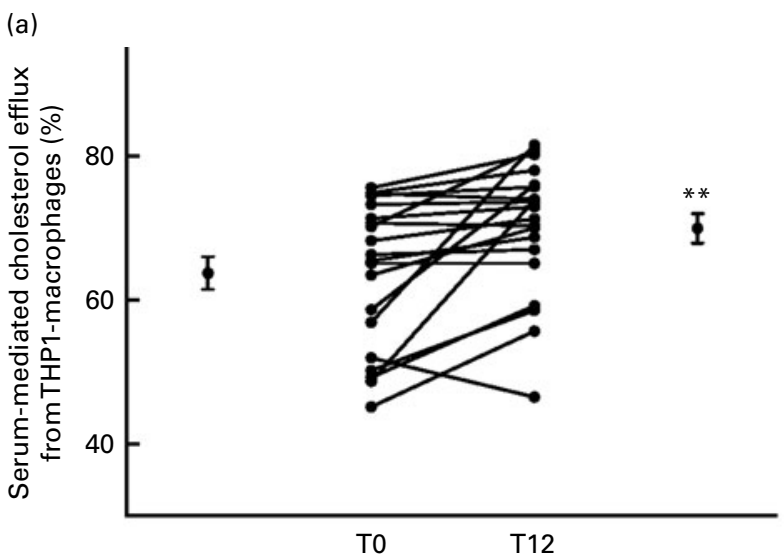

(b)
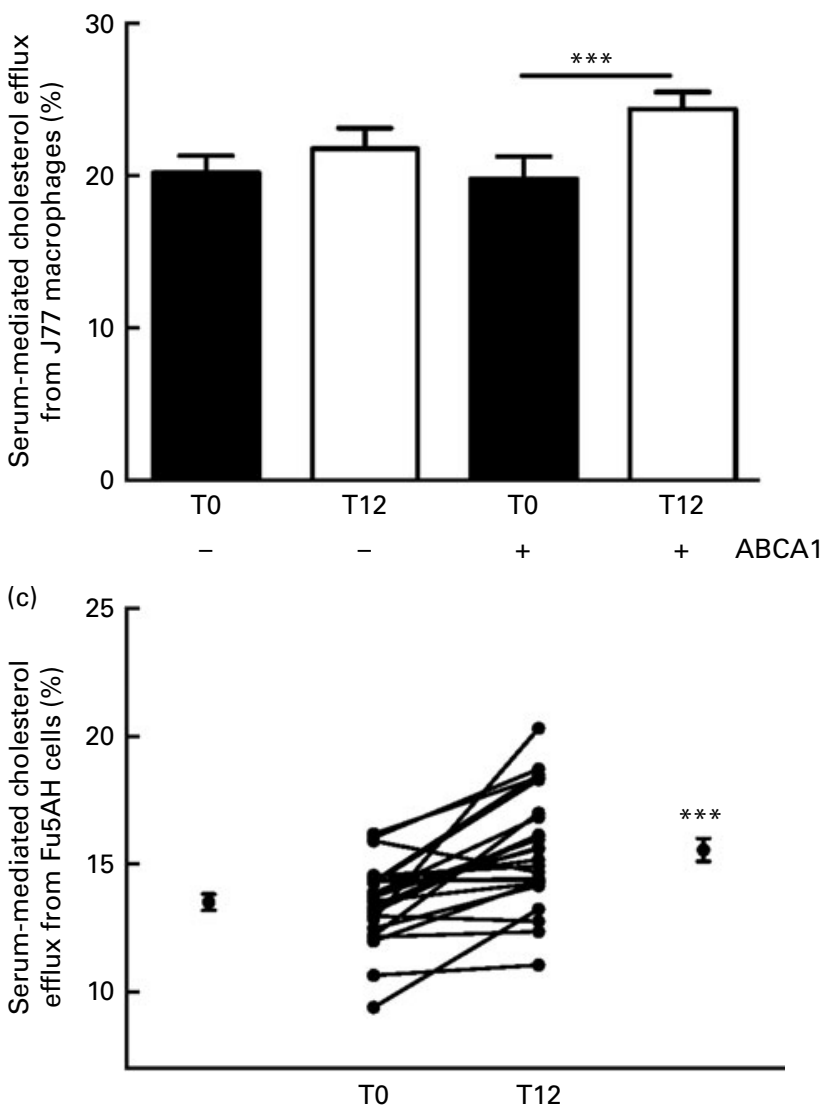

Fig. 1. Extra-virgin olive oil (EVOO) consumption increases serummediated cholesterol efflux. $\left[^{3} \mathrm{H}\right]$ Cholesterol-loaded (a) THP-1 macrophages, (b) ATP-binding cassette (ABC) A1-non-enriched (control) and enriched J774 macrophages and (c) Fu5AH cells were incubated for $24 \mathrm{~h}(4 \mathrm{~h}$ for $\mathrm{J} 774$ macrophages) with $5 \%$ serum. Sera were obtained from volunteers at baseline (T0) and after 12 weeks of EVOO consumption (T12). Cholesterol efflux was determined by liquid scintillation counting, and the percentage of radiolabelled cholesterol efflux was calculated using the following formula: (cpm in medium/cpm in the cell + medium) $\times 100$, where $\mathrm{cpm}$ is counts per min. For each subject, measurements were done in triplicate. A Wilcoxon matched-pairs signed-rank test was used to compare differences between groups. Values are means, with their standard errors represented by vertical bars. Mean values were significantly different from those at T0: ${ }^{\star \star} P<0.01,{ }^{\star \star \star} P<0.001$. macrophages to investigate the involvement of ABCA1. J774 macrophages have a low basal expression of ABCA1, which is overexpressed following a pretreatment with cyclic $\mathrm{AMP}^{(8)}$. It was found that 12 weeks of EVOO consumption increased the capacity of serum to mediate CE from ABCA1-enriched J774 macrophages by $18.85 \%(P<0 \cdot 001)$ while no effect was observed with ABCA1-non-enriched macrophages (Fig. 1(b)).

We used Fu5AH cells to investigate the involvement of the SR-BI pathway in this process. Fu5AH cells express high levels of SR-BI and lack functional $\mathrm{ABCA}^{(5)}$. We found that 12 weeks of EVOO consumption enhanced the capacity of serum to mediate CE from Fu5AH cells via the SR-BI pathway by $14.81 \%(P<0 \cdot 001$; Fig. 1(c)).

\section{Effect of extra-virgin olive oil consumption on HDL-mediated cholesterol efflux}

In light of the results obtained with serum and to better understand the beneficial effect of EVOO on cholesterol homeostasis, we investigated the effect of 12 weeks of EVOO consumption on the capacity of HDL to mediate CE using THP-1 macrophages and Fu5AH cells incubated with whole HDL. We did not use ABCA1-enriched J774 cells because they do not interact with whole HDL or with the $\mathrm{HDL}_{2}$ subfraction $^{(7,37-39)}$. Fig. 2(a) shows that 12 weeks of EVOO consumption enhanced the capacity of whole HDL to mediate CE from THP-1 macrophages by $11.93 \%(P<0 \cdot 01)$.

We then pretreated THP-1 macrophages with 22(R)hydroxy-cholesterol for $24 \mathrm{~h}$ to induce overexpression of ABCG1 ${ }^{(33)}$. mRNA and protein expression measurements confirmed that ABCG1 was up-regulated in THP-1 macrophages (results not shown). While the up-regulation of ABCG1 increased HDL-mediated CE at T0, the increase was significantly higher at T12 (24.57 (SEM 4.89)\% at T12 v. 11.77 (sEm 4.33$) \%$ at T0; Fig. 2(b)).

The effect of 12 weeks of EVOO consumption on the capacity of HDL to mediate CE via the SR-BI pathway was also investigated. Fu5AH cells were used as a cholesterol acceptor. As shown in Fig. 2(c), the capacity of HDL to mediate CE was $16.4 \%$ higher at T12 than at T0 $(P<0.05)$.

To determine which HDL subclass was involved in the increase in $\mathrm{CE}, \mathrm{HDL}_{2}$ and $\mathrm{HDL}_{3}$ isolated from the volunteers at T0 and T12 were incubated separately with $\left[{ }^{3} \mathrm{H}\right]$ cholesterolloaded $\mathrm{Fu} 5 \mathrm{AH}$ cells. $\mathrm{HDL}_{2}$-mediated $\mathrm{CE}$ from Fu5AH cells was $24.1 \%(P<0.05)$ higher at T12 than at T0, while $\mathrm{HDL}_{3}$ had no effect (Fig. 3(a)). In contrast, $\mathrm{HDL}_{3}$-mediated $\mathrm{CE}$ from ABCA1-enriched J774 macrophages was $15.2 \% \quad(P<0.05)$ higher than from control $\mathrm{J} 774$ macrophages (Fig. 3(b)).

The percentage increase in HDL-mediated CE at T12 was comparable to that observed when $5 \%$ serum was used as the cholesterol acceptor.

\section{Effect of extra-virgin olive oil consumption on the distribution of HDL subclasses and their biophysical properties}

We investigated whether the increase in CE through the ABCA1/ABCG1 and SR-BI pathways induced by EVOO 
consumption might be related to a difference in HDL distribution, particularly the levels of pre- $\beta-\mathrm{HDL}_{3}$ and $\alpha-\mathrm{HDL}_{3}$ levels. We found that 12 weeks of EVOO consumption did not induce a significant change in either pre- $\beta$-HDL or $\alpha$-HDL levels (results not shown). We also assessed the distribution of all HDL particles (small $v$. intermediate $v$. large HDL)

(a)

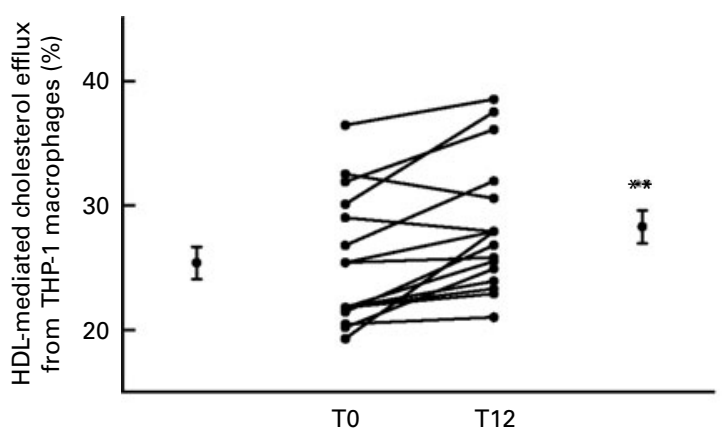

(b)
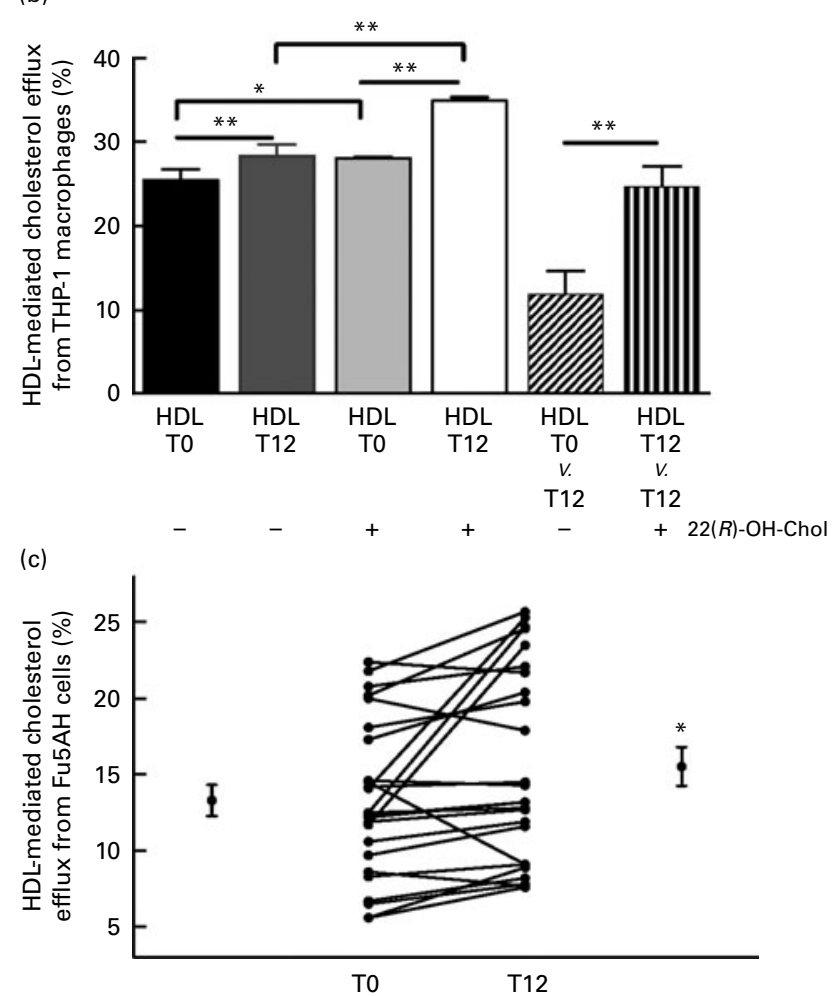

Fig. 2. Extra-virgin olive oil (EVOO) consumption stimulates HDL-mediated cholesterol efflux. (a) THP-1 macrophages were loaded with $\left[{ }^{3} \mathrm{H}\right]$ cholesterol and were then incubated for $24 \mathrm{~h}$ with HDL. (b) $\left.{ }^{3} \mathrm{H}\right]$ Cholesterol-loaded THP-1 macrophages were incubated overnight at $37^{\circ} \mathrm{C}$ without (control) or with $5 \mu \mathrm{M}$-22(R)-hydroxy-cholesterol (22(R)-OH-Chol) to yield ATP-binding cassette $\mathrm{G} 1$-enriched macrophages. After washing, the macrophages were incubated for $24 \mathrm{~h}$ with $\mathrm{HDL}$ to measure cholesterol efflux. (c) Scavenger receptor class $\mathrm{B}$ type I-rich Fu5AH cells were loaded with $\left[{ }^{3} \mathrm{H}\right]$ cholesterol and were then incubated with $\mathrm{HDL}$ for $24 \mathrm{~h}$ to measure cholesterol efflux. All experiments were carried out with $50 \mu \mathrm{g} / \mathrm{ml}$ of $\mathrm{HDL}$ isolated from the plasma of the volunteers at baseline (TO) and after 12 weeks of EVOO consumption (T12). For each subject, measurements were done in triplicate. A Wilcoxon matched-pairs signed-rank test was used to compare differences between groups. Values are means, with their standard errors represented by vertical bars. Mean values were significantly different from those at $\mathrm{T0}:{ }^{*} P<0.05$, ${ }^{\star *} P<0.01$. in an attempt to explain the increase in the capacity of HDL to mediate CE through the ABCG1 and SR-BI pathways. No significant changes were observed between T0 and T12 in the distribution of HDL particles (Table 4).

In addition to the interactions with the ABCA1 transporter and SR-BI receptor, the capacity of HDL to mediate CE also depends on their physico-chemical properties ${ }^{(27)}$, including an increase in the fluidity of the phospholipidic layer of HDL that, in turn, increases the capacity of HDL to mediate CE. Fluidity is an indirect measure of the fatty acid composition of HDL and is determined by anisotropy fluorescence measurements. Due to its high MUFA content, olive oil can induce physico-chemical changes in the lipid composition of HDL and thus in their biophysical structure. The results confirmed that 12 weeks of EVOO consumption increased the fluidity of the phospholipidic layer of HDL by $13 \%$ $(P<0 \cdot 001)$ as measured by fluorescence anisotropy (Fig. 4).
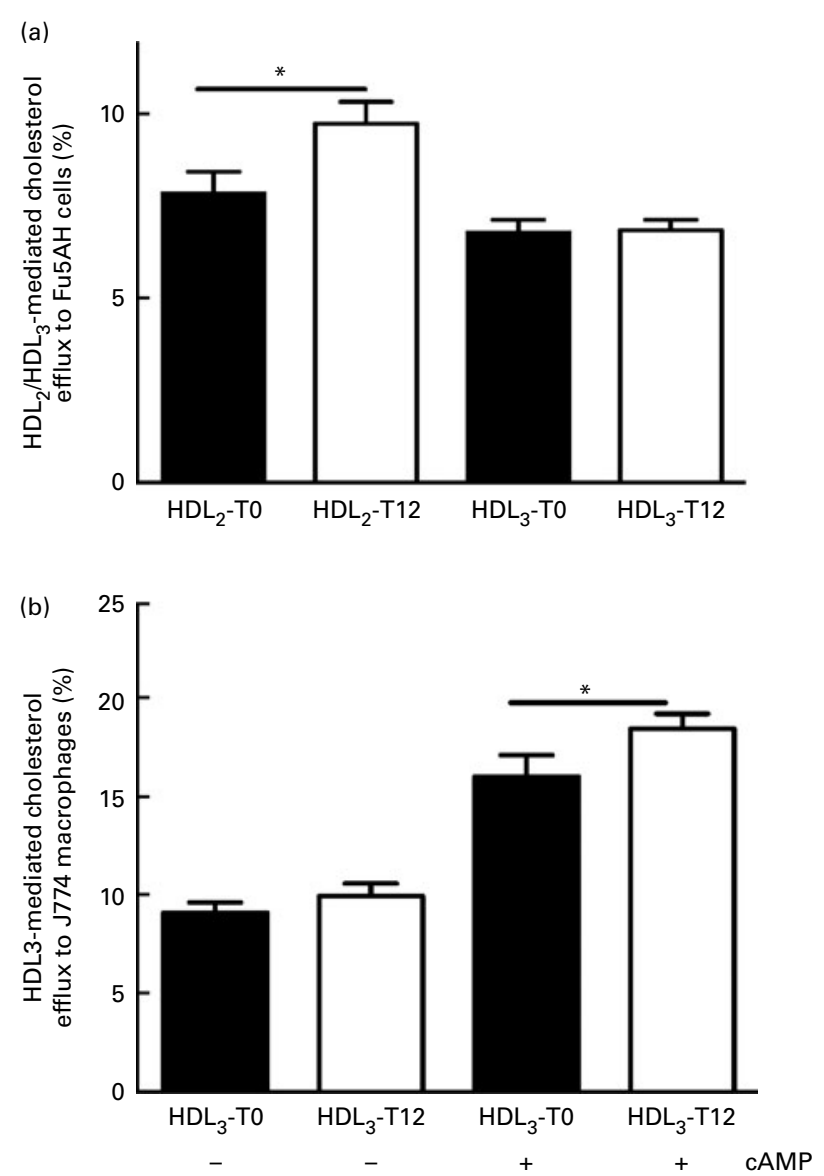

Fig. 3. Extra-virgin olive oil (EVOO) stimulates $\mathrm{HDL}_{2}$ - and $\mathrm{HDL}_{3}$-mediated cholesterol efflux via the scavenger receptor class $B$ type I and ATP-binding cassette (ABC) A1 pathways, respectively. (a) Fu5AH cells were loaded with $\left[{ }^{3} \mathrm{H}\right]$ cholesterol and were then incubated for $24 \mathrm{~h}$ with $50 \mu \mathrm{g} / \mathrm{ml}$ of $\mathrm{HDL}_{2}$ or $\mathrm{HDL}_{3}$. (b) ABCA1-enriched and non-enriched (control) J774 macrophages were incubated with $50 \mu \mathrm{g} / \mathrm{ml}$ of $\mathrm{HDL}_{3}$ for $4 \mathrm{~h}$. $\mathrm{HDL}_{2}$ and $\mathrm{HDL}_{3}$ were isolated from the plasma of the volunteers at baseline (TO) and after 12 weeks of EVOO consumption (T12). For each subject, measurements were done in triplicate. A Wilcoxon matched-pairs signed-rank test was used to compare differences between groups. Values are means, with their standard errors represented by vertical bars. Mean values were significantly different from those at T0: ${ }^{*} P<0 \cdot 05$. cAMP, cyclic AMP. 
Table 4. HDL distribution at baseline (T0) and after 12 weeks of extra-virgin olive oil (EVOO) consumption (T12)*

(Mean values with their standard errors)

\begin{tabular}{|c|c|c|c|c|c|}
\hline \multirow[b]{2}{*}{ HDL distribution } & \multicolumn{2}{|c|}{ T0 } & \multicolumn{2}{|c|}{$\mathrm{T} 12$} & \multirow[b]{2}{*}{$P$} \\
\hline & Mean & sem & Mean & SEM & \\
\hline Large HDL (\%) & 29.17 & 12.04 & $25 \cdot 85$ & $5 \cdot 60$ & 0.55 \\
\hline HDL-1 & 8.47 & $7 \cdot 18$ & $6 \cdot 90$ & 3.03 & 0.63 \\
\hline HDL-2 & $11 \cdot 27$ & $3 \cdot 4$ & $10 \cdot 20$ & $2 \cdot 13$ & 0.52 \\
\hline HDL-3 & 9.42 & 1.72 & 8.73 & 0.93 & 0.40 \\
\hline Intermediate HDL (\%) & 52.08 & 6.33 & 55.93 & 2.29 & 0.19 \\
\hline HDL-4 & 10.78 & 2.47 & $10 \cdot 57$ & 2.04 & 0.87 \\
\hline HDL-5 & $12 \cdot 77$ & 1.30 & 13.67 & 1.19 & 0.23 \\
\hline HDL-6 & 21.55 & $6 \cdot 20$ & $24 \cdot 15$ & 2.59 & 0.36 \\
\hline HDL-7 & 6.95 & $2 \cdot 51$ & 7.58 & $1 \cdot 28$ & 0.59 \\
\hline Small HDL (\%) & 18.75 & 6.03 & $18 \cdot 20$ & 3.64 & 0.85 \\
\hline HDL-8 & 6.68 & 2.33 & $7 \cdot 08$ & 1.30 & 0.72 \\
\hline HDL-9 & 5.40 & 1.71 & 5.40 & 1.02 & 1.00 \\
\hline HDL-10 & $6 \cdot 70$ & $2 \cdot 26$ & $5 \cdot 70$ & 1.69 & 0.2 \\
\hline
\end{tabular}

${ }^{*} \mathrm{~A}$ Wilcoxon matched-pairs signed-rank test was used to compare the differences between T0 and T12.

Effect of extra-virgin olive oil consumption on the capacity of human monocyte-derived macrophages to release excess cholesterol

In the second part of the present study, we investigated the effect of EVOO consumption on the capacity of HMDM to release excess cholesterol, which is the second-limiting step in the RCT process. We used standard human apoA-I as the cholesterol acceptor. Monocytes were isolated from each volunteer at T0 and T12 and were transformed into macrophages (HMDM) as described in the Materials and methods section. HMDM were loaded with $\left[{ }^{3} \mathrm{H}\right]$ cholesterol and were incubated with $25 \mu \mathrm{g} / \mathrm{ml}$ of apoA-I to measure CE. The results revealed that 12 weeks of EVOO consumption increased the

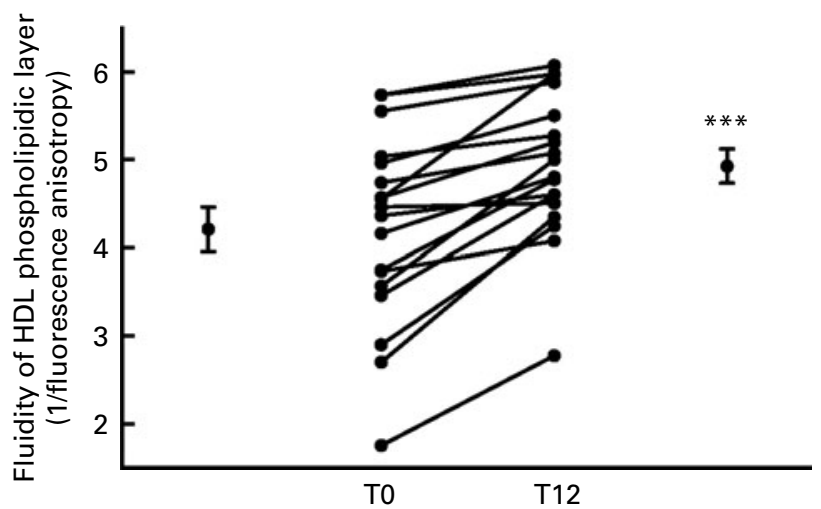

Fig. 4. Extra-virgin olive oil (EVOO) improves HDL phospholipidic layer fluidity. The fluidity of the phospholipidic layer of HDL was measured at baseline (T0) and after 12 weeks of EVOO consumption (T12). Fluidity is the inverse value of anisotropy and is expressed as $1 / r$ (for steady-state fluorescence anisotropy). $r$ was calculated using the following formula: $I_{v}-G I_{p} /\left(I_{v}-2 G I_{p}\right)$, where $I_{v}$ and $I_{\mathrm{p}}$ are the parallel and perpendicular polarised fluorescence intensities, and $G$ is the monochromator grating correction factor. The results were obtained using a 1,6-diphenyl-1,3,5-hexatriene probe and polarisation fluorescence. For each subject, measurements were done in triplicate. A Wilcoxon matched-pairs signed-rank test was used to compare differences between groups. Values are means, with their standard errors represented by vertical bars. ${ }^{* \star}$ Mean values were significantly different from those at T0 $(P<0.001)$. capacity of HMDM to transfer excess cholesterol to apoA-I by over $44 \%(P<0 \cdot 001$; Fig. 5$)$.

In an attempt to explain the increase in cholesterol release from HMDM following EVOO consumption, we measured ABCA1, ABCG1 and SR-BI mRNA and protein expression in HMDM obtained from the volunteers at T0 and T12. As a result, we found that 12 weeks of EVOO consumption increased $A B C A 1$ and $A B C G 1 \mathrm{mRNA}$ expression by $27.51 \%$ $(P<0.0001)$ and $26.48 \%(P<0.001)$, respectively (Fig. 6(a) and (b)). Interestingly, $S R-B I$ mRNA expression was reduced by approximately $30 \%(P<0 \cdot 0001$; Fig. 6(c)). These results were confirmed by the protein expression measurements, which showed that there was a significant increase in ABCA1 $(16.08 \%, P<0.001)$ and ABCG1 $(35.79 \%, P<0.01)$ protein expression and a decrease in SR-BI protein expression $(-2.51 \%, P<0.05)$ following 12 weeks of EVOO consumption (Fig. 6(d) and (e)).

To confirm the effect of EVOO consumption on cholesterol transporter protein levels, especially ABCA1 and ABCG1 in HMDM, we first incubated EVOO polyphenol extracts $(0-320 \mu \mathrm{g} / \mathrm{ml})$ with $\mathrm{J} 774$ macrophages and measured ABCA1 protein expression. Overnight incubation of $\mathrm{J} 774$ macrophages with EVOO polyphenol extracts significantly increased ABCA1 protein expression in a dose-dependent manner (Fig. 7(a)) as measured by Western blot analysis. In light of these results, we then investigated the effect of purified thyrosol and hydroxythyrosol (two major EVOO polyphenols) on ABCA1, ABCG1 and SR-BI protein expression in J774 macrophages. The results (Fig. 7) showed a significant

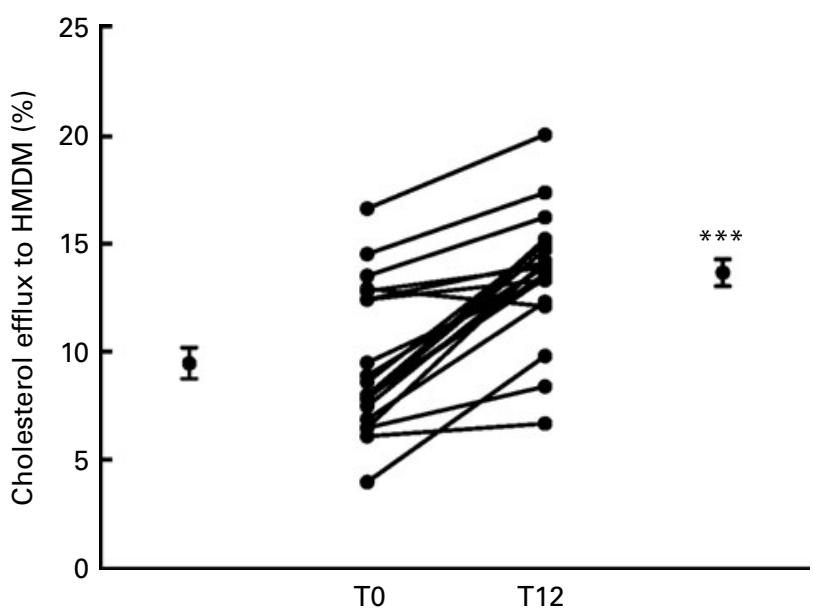

Fig. 5. Extra-virgin olive oil (EVOO) consumption increases the capacity of human monocyte-derived macrophages (HMDM) to release excess cholesterol. Human monocytes were cultured in phorbol myristate acetate-Roswell Park Memorial Institute medium in the presence of autologous sera for 1 week to induce differentiation into macrophages (HMDM). HMDM obtained from the volunteers at baseline (TO) and after 12 weeks of EVOO consumption (T12) were loaded with $\left[{ }^{3} \mathrm{H}\right]$ cholesterol and were then incubated with $25 \mu \mathrm{g} / \mathrm{ml}$ of human apoA-I for $4 \mathrm{~h}$. Cholesterol efflux was determined by liquid scintillation counting, and the percentage of radiolabelled cholesterol released (percentage of cholesterol efflux) was calculated using the following formula: (cpm in medium/cpm in cells + medium) $\times 100$, where $\mathrm{cpm}$ is counts per minute. For each subject, measurements were done in triplicate. A Wilcoxon matched-pairs signed-rank test was used to compare differences between groups. Values are means, with their standard errors represented by vertical bars. ${ }^{* *}$ Mean values were significantly different from those at T0 $(P<0.001)$. 

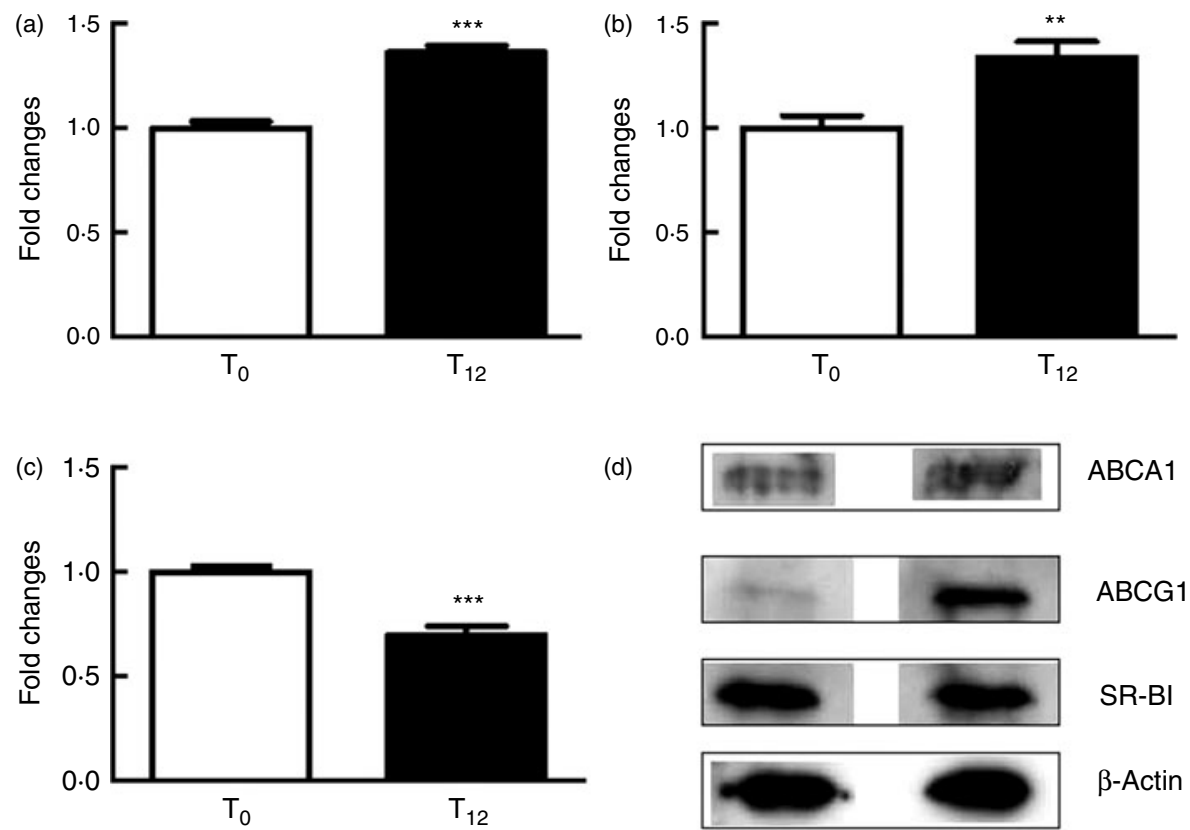

(d)

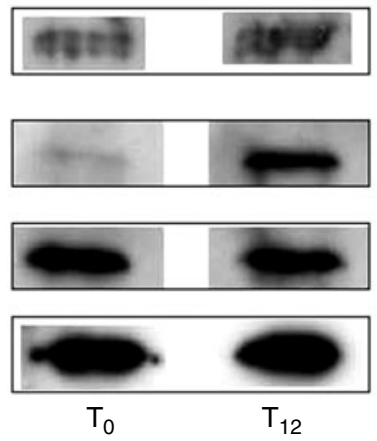

ABCA1

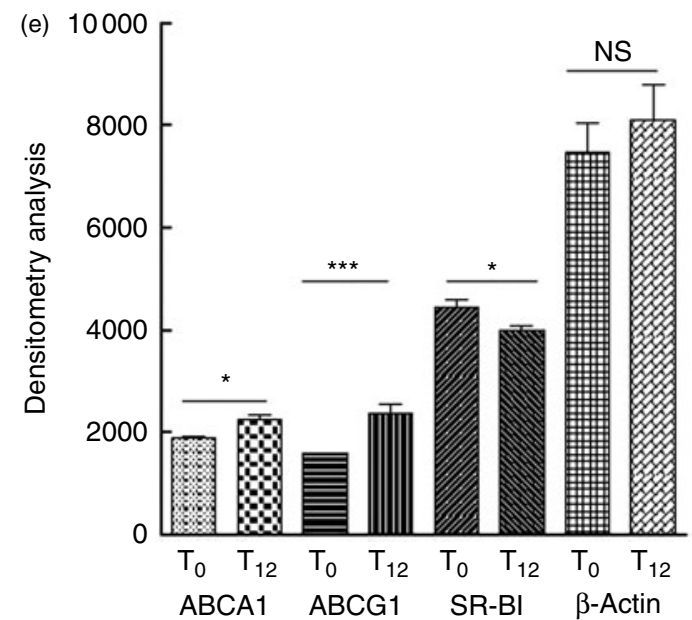

Fig. 6. Extra-virgin olive oil (EVOO) increases ATP-binding cassette (ABC) $A 1$ and $A B C G 1$ mRNA and protein expression in human monocyte-derived macrophages (HMDM). Human monocytes were cultured and differentiated into macrophages in phorbol myristate acetate-Roswell Park Memorial Institute medium in the presence of autologous sera to induce differentiation into macrophages (HMDM). HMDM were lysed and their (a) $A B C A 1$, (b) $A B C G 1$ and (c) scavenger receptor class B type I (SR-BI) mRNA content was quantified by quantitative PCR (qPCR). The comparative threshold cycle $\left(C_{t}\right)$ method was used to quantify the transcript levels and to normalise $\beta$-actin expression. Western blotting was used to evaluate (d) ABCA1, ABCG1 and SR-BI protein levels in HMDM. (e) Protein bands were quantified by densitometry. The qPCR and Western blotting analyses were carried out at baseline (T0) and after 12 weeks of EVOO consumption (T12). For each subject, measurements were done in triplicate. A Wilcoxon matched-pairs signed-rank test was used to compare differences between groups. Values are means, with their standard errors represented by vertical bars. Mean values were significantly different from those at $\mathrm{T0}$ : ${ }^{\star} P<0.05,{ }^{* \star} P<0.01,{ }^{\star \star \star} P<0.001$.

increase in ABCA1 and ABCG1 protein expression in the presence of both tyrosol and hydroxytyrosol $(5$ and $10 \mu \mathrm{M}$; Fig. 7(b), (c), (e) and (f)), whereas no significant effect was observed with respect to SR-BI expression (Fig. 7(d) and (g)).

\section{Discussion}

Olive oil is the main source of fat in the Mediterranean diet. A large body of evidence shows that the Mediterranean diet, which is high in olive oil, is associated with a lower incidence of $\mathrm{CHD}^{(40-43)}$. The beneficial effect of olive oil consumption is related to its high MUFA and phenol content, which have antioxidant and anti-inflammatory properties ${ }^{(19,44-47)}$. A diet rich in olive oil induces a change in lipid metabolism ${ }^{(48)}$ and an increase in the resistance of LDL to lipid peroxidation ${ }^{(49)}$. Covas et al. ${ }^{(19)}$ showed that consuming EVOO increases the level of phenolic compounds in the serum and LDL, which may explain its protective effect against free radical-induced lipid peroxidation. However, no studies have investigated the effect of olive oil consumption by human subjects on the principal anti-atherogenic activity of HDL, which is their capacity to regulate or maintain cholesterol homeostasis.

HDL maintain cholesterol homeostasis by their role in RCT, facilitating the efflux of cholesterol from peripheral tissues and transporting it back to the liver to be eliminated in the bile and faeces $^{(3)}$. This process involves the interaction of lipid-poor 
(a)

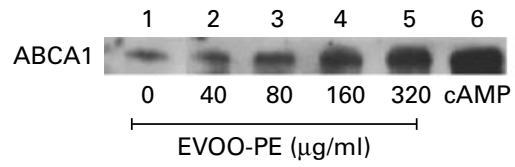

(b)

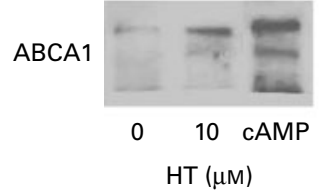

(c)

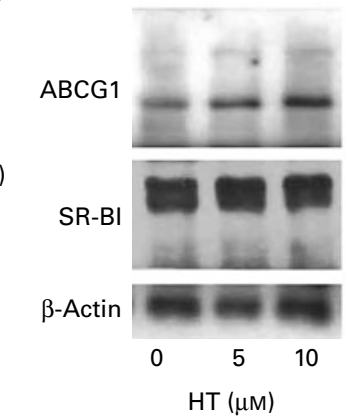

(e)

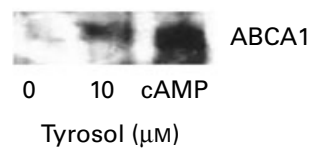

(f)

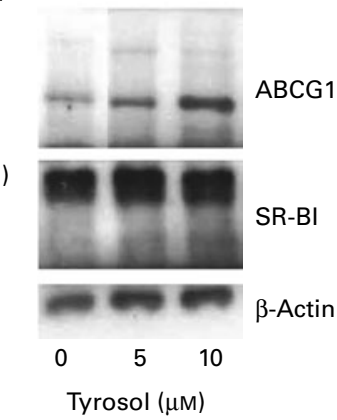

Fig. 7. Extra-virgin olive oil (EVOO) phenolic extracts (EVOO-PE) enhance ATP-binding cassette $(A B C) A 1$ and ABCG1 protein expression in $J 774$ macrophages. J774 macrophages were incubated with various concentrations of EVOO-PE, tyrosol or hydroxytyrosol (HT). After $16 \mathrm{~h}$, the cells were lysed and the lysates were analysed by immunoblotting for $A B C A 1$, ABCG1 and scavenger receptor class B type I (SR-BI) protein expression. (a) ABCA1 expression after incubating J774 macrophages with increasing concentrations of EVOO-PE. Cyclic AMP was used as a control for ABCA1 protein expression. (b) ABCA1, (c) ABCG1 and (d) SR-BI protein expression after incubating $\mathrm{J} 774$ macrophages with hydroxytyrosol. (e) ABCA1, (f) ABCG1 and (g) SR-BI protein expression after incubating J774 macrophages with tyrosol.

apoA-I and mature HDL with ABCA1/ABCG1 transporters and the SR-BI receptor, respectively ${ }^{(50,51)}$; HDL-mediated cholesterol efflux is thus the natural rate-limiting step of $\mathrm{RCT}^{(52)}$. $\mathrm{CE}$ is dependent on apoA-I and HDL concentrations and on the capacity of HDL to mediate the efflux. However, CE from macrophages is also dependent on the expression in the plasma membrane of cholesterol transporters, principally ABCA1 and ABCG $1^{(53)}$. The goals of the present study were to investigate, in healthy volunteers, the effect of 12 weeks of EVOO consumption on the capacity of HDL to mediate $\mathrm{CE}$, and the mechanisms by which EVOO consumption improves cholesterol homeostasis.

The measurements of the capacity of serum to mediate CE revealed that 12 weeks of EVOO consumption significantly stimulated CE from THP-1, J774 and Fu5AH cells (9.8, 23.3 and $15 \cdot 2 \%$, respectively). This can be explained by the fact that serum is a heterogeneous liquid that contains pre- $\beta$ and $\alpha$-migrating HDL, which react with ABCA1/ABCG1 transporters and the SR-BI receptor, respectively. This could be related to physico-chemical changes that occur in HDL following long-term EVOO consumption, which contributes to the formation of smaller HDL particles and greater HDL phospholipidic layer fluidity, and an increase in the linoleic:linolenic acid ratio of $\mathrm{HDL}^{(27)}$. Sola et al. ${ }^{(27,47)}$ reported that a MUFA-

rich diet enhances $\mathrm{HDL}_{3}$-mediated $\mathrm{CE}$ from fibroblast cells, while Sakr et $a l .^{(54)}$ demonstrated that oleic acid-rich fats increase the capacity of serum to promote CE from SR-BIenriched Fu5AH cells and that this effect is correlated with an increase in phospholipid availability. Rosenblat et al. ${ }^{\text {(28) }}$ administered EVOO to apoE-deficient mice and obtained similar results. Efrat et al. ${ }^{(55)}$ showed that EVOO consumption increases di-oleoylphosphatidylcholine (18:1) levels in HDL, which enhances their capacity to mediate CE from macrophages. The increase in phospholipidic layer fluidity is an indirect measure of a change in HDL lipid composition and confirmed that a change in the biochemical composition of HDL following the consumption of EVOO may, in part, contribute to the increase in their capacity to mediate CE. On the other hand, it is well known that the oxidation of HDL significantly affects their capacity to promote $\mathrm{CE}^{(35,56)}$ and that dietary antioxidants increase the resistance of these lipoproteins to lipid peroxidation ${ }^{(47)}$.

While Sola et al. ${ }^{(57)}$ reported that virgin olive oil enrichment increases apoA-I concentrations in high-cardiovascular risk subjects. The present results indicated that EVOO consumption does not affect these parameters in healthy patients. Violante et al. $^{(58)}$ also reported that 3 months of EVOO consumption by hypercholesterolaemic subjects increased serum apoA-I concentrations by about 9\%. However, given the conditions used in the present study, it was not surprising that there was no increase in apoA-I levels after 3 months of EVOO supplementation. On the one hand, all of our volunteers were healthy with no cardiovascular risk factors and, on the other hand, the apoA-I levels and lipid profiles of our volunteers were in normal ranges. In the present study, EVOO had no effect on circulating HDL levels or HDL subclass distribution, but did improve the capacity of HDL to modulate $\mathrm{CE}$, clearly suggesting that EVOO consumption improves HDL functionality, which is more important than HDL concentrations in determining their atheroprotective capacity.

The use of J774, THP-1 and Fu5AH cells allowed us to demonstrate that EVOO consumption stimulates CE mediated by both pre- $\beta-\mathrm{HDL}$ and the mature fractions of $\mathrm{HDL}\left(\mathrm{HDL}_{2}\right.$ and $\mathrm{HDL}_{3}$ ). Pre- $\beta$-HDL are considered important extracellular acceptors of effluxed peripheral tissue cholesterol. They are formed following lipidation of apoA-I by ABCA1. The ABCA1 transporter mediates $\mathrm{CE}$ in a unidirectional manner to apoA-I and pre- $\beta$-HDL. Western blotting and PAGE analyses did not reveal any significant changes in pre- $\beta$-HDL levels or in HDL subclass (small, intermediate and large HDL particles) distribution between T0 and T12. Previous studies, including the present study, showed that oxidative modifications to apoA-I affect its capacity to interact with ABCA1 transporters and to mediate $\mathrm{CE}^{(35,37)}$. While EVOO consumption did not have an effect on apoA-I levels that could explain the increased $\mathrm{CE}$, it should be noted that olive oil contains several components ( $\alpha$-tocopherols, $\beta$-carotene, sterols, terpene, squalene and phenolic compounds ${ }^{(19,41,59)}$ ) with antioxidant activity that may protect apoA-I against oxidative damage, thus contributing to stronger apoA-I/and pre- $\beta$-HDL/ABCA1 interactions that mediate CE. 
The efflux of cholesterol via ABCG1 and SR-BI is mediated by mature HDL, while oxidative damage to apoA-I and whole HDL particles is reduced by polyphenolic compounds, thus increasing CE. The increase in CE mediated by mature HDL also depends on their biochemical and physico-chemical properties, and the increase in the phospholipidic fluidity of HDL may in part explain the improvement in the capacity of HDL to mediate CE.

EVOO consumption increased the capacity of HMDM to release excess cholesterol to apoA-I by $44 \%(P<0 \cdot 001)$, which is comparable to the $42 \%$ increase in CE obtained by Rosenblat et al. ${ }^{(28)}$ with mouse peritoneal macrophages after 8 weeks of EVOO consumption. CE proteins on HMDM plasma membranes, especially ABCA1 and ABCG1 transporter proteins, were significantly up-regulated following EVOO consumption, as measured by mRNA and protein expression. The increase in protein expression may explain the significant increase in cholesterol release from HMDM. A recent study on healthy human subjects showed that consuming coffee, which has a high phenolic acid content, enhances HDL-mediated CE by increasing ABCG1 and SR-BI expression ${ }^{(60)}$. While we cannot explain this discrepancy, it is surprising that polyphenols induced an increase in ABCG1 expression but had no effect on ABCA1 given that both proteins are regulated by the liver $\mathrm{X}$ receptor- $\alpha$, which is activated by phenolic $\operatorname{acids}^{(61)}$, and that, in the present study, phenolic compounds purified from EVOO up-regulated both ABCA1 and ABCG1 transporters in $\mathrm{J} 774$ macrophages.

In contrast, SR-BI mRNA and protein expression was significantly down-regulated after 12 weeks of EVOO consumption. While we have not investigated the factors that may explain this down-regulation, Miles et al. ${ }^{(62)}$ showed that 12 weeks of olive oil consumption by mice caused a reduction in SR-BI protein expression. They interpreted this reduction as a beneficial effect of olive oil consumption in that it prevented the formation of foam cells, which they attributed to unsaturated fatty acids in the olive oil ${ }^{(62)}$. Wang et al. ${ }^{(53)}$ also showed that the SR-BI receptor does not promote macrophage RCT in vivo and that RCT occurs principally via ABCA1 and ABCG1 transporters. We did not observe a significant change in SR-BI expression following $24 \mathrm{~h}$ incubation with purified polyphenols.

It is noteworthy that, in addition to its beneficial effect on the capacity of serum and HDL to mediate CE, 12 weeks of EVOO consumption significantly reduced the total cholesterol:HDL and TAG:HDL ratios. These results are in agreement with those of Violante et al. ${ }^{(58)}$ and showed that EVOO consumption has a beneficial effect on other atherosclerosis markers.

In conclusion, the present results showed that 12 weeks of EVOO consumption significantly increased the capacity of HDL to mediate CE and of HMDM to release excess cholesterol. The increase in the capacity of HDL to mediate CE was independent of HDL concentration and was probably due to improvements in HDL functionality. The increase in the capacity of HMDM to release excess cholesterol can be explained by the increase in ABCA1 and ABCG1 mRNA and protein expression by these cells. The capacity of HDL to mediate CE and of macrophages to release excess cholesterol are two components of RCT, which suggests that EVOO could have a significant effect on the modulation, in vivo, of cholesterol homeostasis and significantly reduce cholesterol deposits in the arteries. Nevertheless, the present study presents some limitations: (1) the design of the study lacks a control group; (2) the diet of the participants was not controlled. Indeed, dietary changes, besides EVOO consumption, could promote an increase in HDL functionality (i.e. other polyphenols or antioxidants); (3) physical activity, which is another possible confounder variable that was not registered; and (4) the age of the participants was in the range of middle-aged/mature people, which limits the extrapolation of the obtained results to a young population. Therefore, due to these limitations, the present study should be considered as a pilot study. However, further studies, considering these limitations, are needed to investigate the effect of EVOO consumption on RCT.

\section{Acknowledgements}

This study was supported by a grant from the Canadian Institutes of Health Research (MOP-89912). The authors' contributions were as follows: A. K. designed the study and obtained funds from the Canadian Institutes of Health Research; O. H. carried out the experiments about the in vivo effect of olive oil; H. B. carried out the experiments on the in vitro effect of EVOO extracts; S. L. participated in the sample collections and the preparation of lipoproteins; O. $\mathrm{H}$. and $\mathrm{H}$. B drafted the manuscript; A. K. revised the manuscript and completed the discussion. All authors read and approved the final manuscript. The authors declare that there are no conflicts of interest.

\section{References}

1. Barter PJ, Caulfield M, Eriksson M, et al. (2007) Effects of torcetrapib in patients at high risk for coronary events. $N$ Engl J Med 357, 2109-2122.

2. Wilson PW, Abbott RD \& Castelli WP (1988) High density lipoprotein cholesterol and mortality. The Framingham Heart Study. Arteriosclerosis 8, 737-741.

3. Glomset JA (1968) The plasma lecithins:cholesterol acyltransferase reaction. J Lipid Res 9, 155-167.

4. Wang N, Lan D, Chen W, et al. (2004) ATP-binding cassette transporters G1 and G4 mediate cellular cholesterol efflux to high-density lipoproteins. Proc Natl Acad Sci U S A 101, 9774-9779.

5. Bortnick AE, Rothblat GH, Stoudt G, et al. (2000) The correlation of ATP-binding cassette 1 mRNA levels with cholesterol efflux from various cell lines. J Biol Chem 275, 28634-28640.

6. Ruano J, Lopez-Miranda J, Fuentes F, et al. (2005) Phenolic content of virgin olive oil improves ischemic reactive hyperemia in hypercholesterolemic patients. J Am Coll Cardiol 46, $1864-1868$.

7. Tall AR, Costet P \& Wang N (2002) Regulation and mechanisms of macrophage cholesterol efflux. J Clin Invest 110, 899-904.

8. Yancey PG, Bortnick AE, Kellner-Weibel G, et al. (2003) Importance of different pathways of cellular cholesterol efflux. Arterioscler Thromb Vasc Biol 23, 712-719. 
9. Smith JD (2006) Insight into ABCG1-mediated cholesterol efflux. Arterioscler Thromb Vasc Biol 26, 1198-1200.

10. Favari E, Lee M, Calabresi L, et al. (2004) Depletion of prebeta-high density lipoprotein by human chymase impairs ATP-binding cassette transporter A1- but not scavenger receptor class B type I-mediated lipid efflux to high density lipoprotein. J Biol Chem 279, 9930-9936.

11. Baldan A, Bojanic DD \& Edwards PA (2009) The ABCs of sterol transport. J Lipid Res 50, S80-S85.

12. Yancey PG, de la Llera-Moya M, Swarnakar S, et al. (2000) High density lipoprotein phospholipid composition is a major determinant of the bi-directional flux and net movement of cellular free cholesterol mediated by scavenger receptor BI. J Biol Chem 275, 36596-36604.

13. Brunner E \& Iso H (2008) Fish oil and secondary prevention of cardiovascular disease. BMJ 337, a2541.

14. Ordovas JM, Kaput J \& Corella D (2007) Nutrition in the genomics era: cardiovascular disease risk and the Mediterranean diet. Mol Nutr Food Res 51, 1293-1299.

15. Hu FB, Stampfer MJ, Manson JE, et al. (1997) Dietary fat intake and the risk of coronary heart disease in women. $N$ Engl J Med 337, 1491-1499.

16. Visioli F, Poli A \& Gall C (2002) Antioxidant and other biological activities of phenols from olives and olive oil. Med Res Rev 22, 65-75.

17. Lopez-Miranda J, Perez-Jimenez F, Ros E, et al. (2010) Olive oil and health: summary of the II international conference on olive oil and health consensus report, Jaen and Cordoba (Spain) 2008. Nutr Metab Cardiovasc Dis 20, 284-294.

18. Castaner O, Fito M, Lopez-Sabater MC, et al. (2011) The effect of olive oil polyphenols on antibodies against oxidized LDL. A randomized clinical trial. Clin Nutr 30, 490-493.

19. Covas MI, de la Torre K, Farre-Albaladejo M, et al. (2006) Postprandial LDL phenolic content and LDL oxidation are modulated by olive oil phenolic compounds in humans. Free Radic Biol Med 40, 608-616.

20. Aviram M \& Eias K (1993) Dietary olive oil reduces lowdensity lipoprotein uptake by macrophages and decreases the susceptibility of the lipoprotein to undergo lipid peroxidation. Ann Nutr Metab 37, 75-84.

21. Nicolaiew N, Lemort N, Adorni L, et al. (1998) Comparison between extra virgin olive oil and oleic acid rich sunflower oil: effects on postprandial lipemia and LDL susceptibility to oxidation. Ann Nutr Metab 42, 251-260.

22. Aguilera CM, Mesa MD, Ramirez-Tortosa MC, et al. (2004) Sunflower oil does not protect against LDL oxidation as virgin olive oil does in patients with peripheral vascular disease. Clin Nutr 23, 673-681.

23. Rodenas S, Rodriguez-Gil S, Merinero MC, et al. (2005) Dietary exchange of an olive oil and sunflower oil blend for extra virgin olive oil decreases the estimate cardiovascular risk and LDL and apolipoprotein AII concentrations in postmenopausal women. J Am Coll Nutr 24, 361-369.

24. Perez-Jimenez F, Espino A, Lopez-Segura F, et al. (1995) Lipoprotein concentrations in normolipidemic males consuming oleic acid-rich diets from two different sources: olive oil and oleic acid-rich sunflower oil. Am J Clin Nutr 62, 769-775.

25. Perona JS, Canizares J, Montero E, et al. (2004) Virgin olive oil reduces blood pressure in hypertensive elderly subjects. Clin Nutr 23, 1113-1121.

26. Movva R \& Rader DJ (2008) Laboratory assessment of HDL heterogeneity and function. Clin Chem 54, 788-800.

27. Sola R, Motta C, Maille M, et al. (1993) Dietary monounsaturated fatty acids enhance cholesterol efflux from human fibroblasts. Relation to fluidity, phospholipid fatty acid composition, overall composition, and size of HDL3. Arterioscler Thromb 13, 958-966.

28. Rosenblat M, Volkova N, Coleman R, et al. (2008) Antiatherogenicity of extra virgin olive oil and its enrichment with green tea polyphenols in the atherosclerotic apolipoprotein-E-deficient mice: enhanced macrophage cholesterol efflux. J Nutr Biochem 19, 514-523.

29. Khallouki F, Younos C, Soulimani R, et al. (2003) Consumption of argan oil (Morocco) with its unique profile of fatty acids, tocopherols, squalene, sterols and phenolic compounds should confer valuable cancer chemopreventive effects. Eur J Cancer Prev 12, 67-75.

30. Ruiz-Gutierrez V, Juan ME, Cert A, et al. (2000) Determination of hydroxytyrosol in plasma by HPLC. Anal Chem 72, 4458-4461.

31. Sattler W, Mohr D \& Stocker R (1994) Rapid isolation of lipoproteins and assessment of their peroxidation by highperformance liquid chromatography postcolumn chemiluminescence. Methods Enzymol 233, 469-489.

32. Chinetti G, Griglio S, Antonucci M, et al. (1998) Activation of proliferator-activated receptors alpha and gamma induces apoptosis of human monocyte-derived macrophages. $J$ Biol Chem 273, 25573-25580.

33. Kaplan R, Gan X, Menke JG, et al. (2002) Bacterial lipopolysaccharide induces expression of ABCA1 but not ABCG1 via an LXR-independent pathway. J Lipid Res 43, 952-959.

34. Apostolou F, Gazi IF, Kostoula A, et al. (2009) Persistence of an atherogenic lipid profile after treatment of acute infection with Brucella. J Lipid Res 50, 2532-2539.

35. Berrougui H, Isabelle M, Cloutier M, et al. (2007) Age-related impairment of HDL-mediated cholesterol efflux. J Lipid Res 48, 328-336.

36. Beck R, Bertolino S, Abbot SE, et al. (1998) Modulation of arachidonic acid release and membrane fluidity by albumin in vascular smooth muscle and endothelial cells. Circ Res 83, 923-931.

37. Shao B, Tang C, Heinecke JW, et al. (2010) Oxidation of apolipoprotein A-I by myeloperoxidase impairs the initial interactions with ABCA1 required for signaling and cholesterol export. J Lipid Res 51, 1849-1858.

38. Waddington EI, Boadu E \& Francis GA (2005) Cholesterol and phospholipid efflux from cultured cells. Methods 36, 196-206.

39. Wang N, Silver DL, Costet P, et al. (2000) Specific binding of ApoA-I, enhanced cholesterol efflux, and altered plasma membrane morphology in cells expressing ABC1. J Biol Chem 275, 33053-33058.

40. Keys A, Menotti A, Karvonen MJ, et al. (1986) The diet and 15 -year death rate in the seven countries study. Am J Epidemiol 124, 903-915.

41. Konstantinidou V, Covas MI, Munoz-Aguayo D, et al. (2010) In vivo nutrigenomic effects of virgin olive oil polyphenols within the frame of the Mediterranean diet: a randomized controlled trial. FASEB J 24, 2546-2557.

42. Covas MI (2007) Olive oil and the cardiovascular system. Pharmacol Res 55, 175-186.

43. Estruch R, Martinez-Gonzalez MA, Corella D, et al. (2006) Effects of a Mediterranean-style diet on cardiovascular risk factors: a randomized trial. Ann Intern Med 145, 1-11.

44. Bogani P, Galli C, Villa M, et al. (2007) Postprandial antiinflammatory and antioxidant effects of extra virgin olive oil. Atherosclerosis 190, 181-186.

45. de la Torre-Carbot K, Chavez-Servin JL, Jauregui O, et al. (2010) Elevated circulating LDL phenol levels in men who consumed virgin rather than refined olive oil are associated with less oxidation of plasma LDL. J Nutr 140, 501-508. 
46. Weinbrenner T, Fito M, de la Torre R, et al. (2004) Olive oils high in phenolic compounds modulate oxidative/antioxidative status in men. J Nutr 134, 2314-2321.

47. Sola R, La Ville AE, Richard JL, et al. (1997) Oleic acid rich diet protects against the oxidative modification of high density lipoprotein. Free Radic Biol Med 22, 1037-1045.

48. Chan YM, Demonty I, Pelled D, et al. (2007) Olive oil containing olive oil fatty acid esters of plant sterols and dietary diacylglycerol reduces low-density lipoprotein cholesterol and decreases the tendency for peroxidation in hypercholesterolaemic subjects. Br J Nutr 98, 563-570.

49. Mata P, Varela O, Alonso R, et al. (1997) Monounsaturated and polyunsaturated $n-6$ fatty acid-enriched diets modify LDL oxidation and decrease human coronary smooth muscle cell DNA synthesis. Arterioscler Thromb Vasc Biol 17, 2088-2095.

50. Wang N, Silver DL, Thiele C, et al. (2001) ATP-binding cassette transporter A1 (ABCA1) functions as a cholesterol efflux regulatory protein. J Biol Chem 276, 23742-23747.

51. Kennedy MA, Barrera GC, Nakamura K, et al. (2005) ABCG1 has a critical role in mediating cholesterol efflux to HDL and preventing cellular lipid accumulation. Cell Metab 1, $121-131$.

52. Fu Y (2010) Rate-limiting factors of cholesterol efflux in reverse cholesterol transport: acceptors and donors. Clin Exp Pharmacol Physiol 37, 703-709.

53. Wang X, Collins HL, Ranalletta M, et al. (2007) Macrophage ABCA1 and ABCG1, but not SR-BI, promote macrophage reverse cholesterol transport in vivo. J Clin Invest 117, 2216-2224

54. Sakr SW, Senault C, Vacher D, et al. (1996) Oleic acid-rich fats increase the capacity of postprandial serum to promote cholesterol efflux from Fu5AH cells. Biochim Biophys Acta 1300, 49-55.

55. Efrat M, Rosenblat M, Mahmood S, et al. (2009) Di-oleoyl phosphatidylcholine (PC-18:1) stimulates paraoxonase 1 (PON1) enzymatic and biological activities: in vitro and in vivo studies. Atherosclerosis 202, 461-469.

56. Bonnefont-Rousselot D, Motta C, Khalil AO, et al. (1995) Physicochemical changes in human high-density lipoproteins (HDL) oxidized by gamma radiolysis-generated oxyradicals. Effect on their cholesterol effluxing capacity. Biochim Biophys Acta 1255, 23-30.

57. Sola R, Fito M, Estruch R, et al. (2011) Effect of a traditional Mediterranean diet on apolipoproteins B, A-I, and their ratio: a randomized, controlled trial. Atherosclerosis 218, 174-180.

58. Violante B, Gerbaudo L, Borretta G, et al. (2009) Effects of extra virgin olive oil supplementation at two different low doses on lipid profile in mild hypercholesterolemic subjects: a randomised clinical trial. J Endocrinol Invest 32, 794-796.

59. Cabello-Moruno R, Perona JS, Osada J, et al. (2007) Modifications in postprandial triglyceride-rich lipoprotein composition and size after the intake of pomace olive oil. $J$ Am Coll Nutr 26, 24-31.

60. Uto-Kondo H, Ayaori M, Ogura M, et al. (2010) Coffee consumption enhances high-density lipoprotein-mediated cholesterol efflux in macrophages. Circ Res 106, 779-787.

61. Sevov M, Elfineh L \& Cavelier LB (2006) Resveratrol regulates the expression of LXR-alpha in human macrophages. Biochem Biophys Res Commun 348, 1047-1054.

62. Miles EA, Wallace FA \& Calder PC (2001) An olive oil-rich diet reduces scavenger receptor mRNA in murine macrophages. Br J Nutr 85, 185-191. 